\title{
A QUESTION OF VAN DEN DRIES AND A THEOREM OF LIPSHITZ AND ROBINSON; NOT EVERYTHING IS STANDARD
}

\author{
EHUD HRUSHOVSKI AND YA'ACOV PETERZIL
}

\begin{abstract}
We use a new construction of an o-minimal structure, due to Lipshitz and Robinson, to answer a question of van den Dries regarding the relationship between arbitrary o-minimal expansions of real closed fields and structures over the real numbers. We write a first order sentence which is true in the Lipshitz-Robinson structure but fails in any possible interpretation over the field of real numbers.
\end{abstract}

An o-minimal structure is by definition an expansion $\mathcal{M}$ of a linear ordering, such that every definable subset of the linear ordering is a finite union of intervals whose end points are in $\mathcal{M} \cup\{ \pm \infty\}$.

Although o-minimal expansions of discrete linear orderings do exist (e.g. $\langle\mathbb{Z},<, z \mapsto z+1\rangle$ ), these were recognized early on to have a relatively poor structure and therefore, in the above definition, one often assumes that the linear ordering is dense without endpoints.

As was shown in [5], an o-minimal structures is, at least locally, one of the following three possibilities: It is degenerate (basically an expansion of the linear ordering by unary functions), an (interval in an) ordered vector space over an ordered division ring, or an expansion of a real closed field.

Since ordered vector spaces over noncommutative ordered division rings essentially cannot be further expanded while preserving o-minimality (see [5]), it is within the third possibility, of expansions of real closed fields, where new o-minimal structures can be found. Indeed, construction of new o-minimal structures is usually carried out in this context, and much work has been done in this direction in the past twenty years. However, until very recently, all of these new o-minimal structures were expansions of the field of real numbers $\langle\mathbb{R},<,+, \cdot\rangle$.

Of course, we know from model theory that every such structure $\mathcal{M}$ over $\mathbb{R}$ gives rise to an o-minimal expansion $\mathcal{M}^{*}$ of a nonstandard real closed field. Now let $\mathcal{M}_{1}^{*}$ be a structure with the same universe as $M^{*}$, and whose relations are some of those definable with parameters in $M^{*}$. $\mathcal{M}_{1}^{*}$ may not be elementarily equivalent to any structure over the real 
numbers, for the simple reason that it may contain new constants for "infinitely large" elements.

A question arises. How can we recognize that the theory of $\mathcal{M}_{1}^{*}$ has begun its model theoretic life as a theory of a structure over the real numbers? And in general, is it possible that every o-minimal expansion of a real closed field arises in this way?

Here is one possibility, suggested by L. van den Dries in [2], for a precise formulation to the above question:

Let $\phi\left(R_{1}, \ldots, R_{n}, f_{1}, \ldots, f_{k}\right)$ be a sentence in a language $\mathcal{L}$ expanding the language of ordered rings, with $R_{1}, \ldots, R_{n}, f_{1}, \ldots, f_{k}$ all relation and function symbols that are different than $\{<,+, \cdot\}$.

$\left.{ }^{*}\right) \quad$ Assume that $\mathcal{N}$ is an o-minimal expansion of a real closed field in the language $\mathcal{L}$ such that $\mathcal{N} \models \phi\left(R_{1}, \ldots, R_{n}, f_{1}, \ldots, f_{k}\right)$. Does it follow that:

$$
\langle\mathbb{R},<,+, \cdot\rangle \models \exists R_{1}, \ldots, R_{n} \exists f_{1}, \ldots, f_{k} \phi\left(R_{1}, \ldots, R_{n}, f_{1}, \ldots, f_{k}\right) ?
$$

Notice that when $\mathcal{N}$ equals $\mathcal{M}_{1}^{*}$ from the above example then indeed the answer is positive.

For a given $\phi$ let us denote by $(* *)$ the contra-positive to the above. Namely the following transfer question:

(**) If $\phi\left(R_{1}, \ldots, R_{n}, f_{1}, \ldots, f_{k}\right)$ is true in every possible expansion of $\langle\mathbb{R},<,+, \cdot\rangle$ then is it necessarily true in every o-minimal expansion of a real closed field (in the language $\mathcal{L}$ )?

Remark As van den Dries points out, one gets a negative answer to the above questions, if the real closed field assumption is omitted. Indeed, let $\phi$ be the statement: "If $\lambda_{1} \lambda_{2}$ are two continuous automorphisms of $\langle\mathbb{R},+\rangle$ then $\lambda_{1} \lambda_{2}=\lambda_{2} \lambda_{1}$ ". This is true in every interpretation of $\lambda_{1}, \lambda_{2}$ over the ordered group of the reals but fails in ordered vector spaces over noncommutative ordered division rings.

Here are some instances of sentences $\phi$ for which $\left(^{* *}\right)$ was (nontrivially) shown to have a positive answer:

1. (Invariance of Domain): If $f$ is a continuous injective function from an open set in $\mathbb{R}^{n}$ into $\mathbb{R}^{n}$ then it is an open map ([7]).

2. If $G$ is a closed and bounded subset of $R^{n}$ and $\langle G, \star\rangle$ is a topological group then $G$ has a torsion point (see [3] for a precise count of the torsion points).

3. If $F$ is a function from the open unit disc in $\mathbb{C}$ into $\mathbb{C}$ which is differentiable (with respect to $\mathbb{C}$ ) then its derivative $F^{\prime}(z)$ is differentiable as well $([\underline{6})$. 
It has already been pointed out that a positive answer to $(*)$ would yield some strong consequences. E.g., in [1, Berarducci and Servi showed that the decidability of the real exponential field would follow. It would also have trivialized a significant part of the theory of groups in o-minimal structures. On the other hand, a negative answer required a new procedure for constructing o-minimal expansions of real closed fields different than the real numbers and until recently none was known.

This has changed with the work of Lipshitz and Robinson (4]), where they constructed the following new o-minimal expansion of a real closed field:

The underlying real closed field $R$ is the field of Puiseux series in $t$ over $\mathbb{R}$,

$$
R=\bigcup_{n} \mathbb{R}\left(\left(t^{1 / n}\right)\right),
$$

with $t$ an infinitesimal in the ordering of the field. If $p\left(\xi_{1}, \ldots, \xi_{n}\right)$ is a formal power series over $\mathbb{R}$ (the ring of all such power series is denoted by $\left.\mathbb{R}\left[\left[\xi_{1}, \ldots, \xi_{n}\right]\right]\right)$ then $p\left(\xi_{1}, \ldots, \xi_{n}\right)$ converges on the infinitesimal cube $[-t, t]^{n} \subseteq R^{n}$. Denote by $f_{p}\left(x_{1}, \ldots, x_{n}\right)$ the corresponding function, which is set to be zero outside the box $[-t, t]^{n}$. The theorem of Lipshitz and Robinson says:

Theorem 1. The structure $\mathcal{M}=\left\langle R, f_{p}\right\rangle_{p \in \mathbb{R}\left[\left[\xi_{1}, \ldots, \xi_{n}\right]\right]}$ is o-minimal.

Here are some basic properties:

Fact 2. (1) The map $p \mapsto f_{p}$ is an embedding of rings from the ring $\mathbb{R}\left[\left[\xi_{1}, \ldots, \xi_{n}\right]\right]$ into the ring of definable functions on $[-t, t]^{n}$.

(2) For $p \in \mathbb{R}\left[\left[\xi_{1}, \ldots, \xi_{n}\right]\right]$, and $q=\partial p / \partial x_{i}$ the formal derivative with respect to $\xi_{i}$, we have $\partial f_{p} / \partial x_{i}=f_{q}$ on $-[t, t]^{n}$, where the partial derivative of $f_{p}$ is taken with respect to the real closed field $R$.

Proof (1) is standard. For (2), consider for simplicity the 1-variable case. Then, it follows from (1), that for small $x$ and $h \neq 0$,

$$
\frac{f_{p}(x+h)-f_{p}(x)}{h}=f_{\frac{p(\xi+h)-p(\xi)}{h}}(x) .
$$

The result easily follows.

Denote by $K=R(\sqrt{-1})$ the algebraic closure of $R$, identified as usual with $R^{2}$. Notice that the product topology induced on $K$ from $R$ is the same as the valuation topology when we identify $K$ with the field of Puiseux series over $\mathbb{C}$. We denote by $D \subseteq K$ the open disc around of radius $t$ (inthe sense of $R^{2}$ ) around $0 \in K$. As in [6], we say 
that an $\mathcal{M}$-definable function $F$ from an open set $U \subseteq K$ into $K$ is $K$-differentiable at $z_{0} \in U$ if the limit

$$
\lim _{z \rightarrow 0} \frac{F\left(z_{0}+z\right)-F\left(z_{0}\right)}{z}
$$

exists in $K$. We call this limit $F^{\prime}\left(z_{0}\right)$. Notice that the definition through limits gives a formula $\psi_{\text {der }}\left(F, z_{0}\right)$ in the language of ordered rings, augmented by a symbol for $F$ (or more precisely by two functions symbols for the real and imaginary parts of $F$ ), such that for any structure $\langle\mathbb{R},<,+, \cdot, F\rangle$ and any $z_{0} \in \mathbb{C}$, the function $F(z)$ is complex differentiable at $z_{0}$ if and only if $\psi_{\text {der }}\left(F, z_{0}\right)$ holds.

We now return to our field of Puiseux series $R$ and its algebraic closure $K$. If $p(\zeta)$ is a formal power series in $\mathbb{R}[[\zeta]]$ then $p(z)$ converges, in the topology induced on $K$ from $R$, for all $z \in D \subseteq K$. Let $F_{p}(z)$ be the corresponding function from $D$ into $K$. Notice that $F_{p}$ as two coordinate functions $\left(f_{p_{1}}, f_{p_{2}}\right)(x, y)$, where $p_{1}, p_{2}$ are themselves power series in $\mathbb{R}\left[\left[\xi_{1}, \xi_{2}\right]\right]$ (indeed, this follows from the fact that each map $z \mapsto z^{n}$ can be written, in $R$-coordinates, as $\left(q_{1}(x, y), q_{2}(x, y)\right)$ where $q_{1}$ and $q_{2}$ are homogeneous polynomials of degree $n$ ). Therefore, the function $F_{p}(z)$ is definable in $\mathcal{M}$.

Fact 3. (1) The map $p \mapsto f_{p}$ is an embedding of rings from the ring $\mathbb{R}[[\zeta]]$ into the ring of definable functions from $D$ into $K$.

(2) For $p \in \mathbb{R}[[\zeta]]$, the function $F_{p}(z)$ is $K$-differentiable. If $p^{\prime}(\zeta)$ is the formal derivative with respect to $\zeta$, we have $F_{p}^{\prime}(z)=F_{p^{\prime}}(z)$ for all $z$ in $D$.

Proof The proof of (1) follows from Clause (1) in Fact 2 . The proof of (2) is just like the proof of Clause (2) above.

We can now produce a sentence $\phi$ for which the answer to $\left(^{*}\right)$ is negative: The signature contains two 2-ary function symbols $f_{1}$ and $f_{2}$.

Let $\phi\left(f_{1}, f_{2}\right)$ be the following sentence (using $F(z)$ for simplification instead of $\left.\left(f_{1}, f_{2}\right)(x, y)\right)$ :

There exists $r>0$, such that for all $z=x+$ iy, if $|z|<r$ then $\psi_{\text {der }}(F, z)$ and

$$
F(z)=z^{2} F^{\prime}(z)+z
$$

Fact 4. (1) Let $p(\zeta)=\Sigma_{n=1}^{\infty}(n-1) ! \zeta^{n}$. Then $F_{p}(z)$ is a solution to 3.1 on some open neighborhood of $0 \in K$.

(2) The sentence $\phi\left(f_{1}, f_{2}\right)$ is false in $\left\langle\mathbb{R},<,+, \cdot, f_{1}, f_{2}\right\rangle$ for every possible interpretation of $f_{1}, f_{2}$. 
Proof (1) It is easy to verify that $p(\zeta)$ is a formal solution to (3.1). It follows from Fact 3 that $F_{p}$ is a solution to the same equation on $D$.

For (2), notice that if $F(z)$ were a complex differentiable function which solves 3.1 then its Taylor series at 0 must be $p(\zeta)$. However, this power series is divergent at every nonzero $z \in \mathbb{C}$.

The sentence $\phi\left(f_{1}, f_{2}\right)$ is therefore true in $\mathcal{M}$, with $f_{1}^{\mathcal{M}}, f_{2}^{\mathcal{M}}$ interpreted as the real and imaginary parts of $F_{p}$ (for $p$ as in the last Fact), but fails over the real numbers in every possible interpretation of $f_{1}, f_{2}$.

A question Because of its strong potential consequences we are tempted to re-formulate van den Dries' original question as follows:

Find a restricted class $\mathcal{K}$ of o-minimal expansions of real closed fields such that every finite theory $T$ in a language $\mathcal{L}$ expanding the langauge of real closed fields, which holds in some o-minimal expansion of a real closed field, has an interpretation in one of the structures in $\mathcal{K}$.

Another possible variation on the requirement from the above $\mathcal{K}$ is the following local version:

Consider a sentence $\phi$ in a language $\mathcal{L}$ expanding real closed fields, and an o-minimal $\mathcal{L}$-structure $\mathcal{M}$. For every $t>0$ in $\mathcal{M}$, consider the sentence $\phi_{t}$ obtained from $\phi$ by restricting all quantifiers and relations to cartesian products of $[-t, t]$. By o-minimality, the truth value of $\phi_{t}$ in $\mathcal{M}$ stabilizes as as $t$ approaches 0 . We ask: Given $\phi$ and $\mathcal{M}$, is there a structure $\mathcal{M}_{\phi}$ in $\mathcal{K}$ and an interpretation of all the symbols in $\phi$ in $\mathcal{M}_{\phi}$ such that the limit truth value of $\phi_{t}$ is the same in $\mathcal{M}$ and in $\mathcal{M}_{\phi}$ ?

We feel that the Lipshitz-Robinson model described above could play a significant role in finding an appropriate $\mathcal{K}$.

We first realized that an equation such as 3.1 should exist by considering Sofia Kovalevskaya's example of an analytic differential equation whose solutions are not analytic. The actual example in this paper was arrived at after a series of simplifications and corrections.

\section{REFERENCES}

[1] Alessandro Berarducci and Tamara Servi, An effective version of Wilkie's theorem of the complement and some effective o-minimality results, Ann. Pure Appl. Logic 125 (2004), 43-74.

[2] Lou van den Dries, o-minimal structures, Logic: from Foundations to Applications (Staffordshire, 1993), Oxford Sci. Publ., Oxford Univ. Press, New York, 1996, pp. 137-185.

[3] Mário J. Edmundo and Margarita Otero, Definably compact abelian groups, J. Math. Log. 4 (2004), 163-180.

[4] Leonard Lipshitz and Zachary Robinson, Overconvergent real closed quantifier elimination. 
[5] Ya'acov Peterzil and Sergei Starchenko, A trichotomy theorem for o-minimal structures, Proc. London Math. Soc. (3) 77 (1998), 481-523.

[6] _ Expansions of algebraically closed fields in o-minimal structures, Selecta Math. (N.S.) 7 (2001), 409-445.

[7] A. Woerheide, O-minimal homology, Ph.D. Thesis, University of Illinois at Urbana-Champaign (1996).

Department of Mathematics, Hebrew University, Jerusalem, ISRAel

E-mail address: ehud@math.huji.ac.il

Department of Mathematics, University of Haifa, Haifa, ISRAEL

E-mail address: kobi@math.haifa.ac.il 